\title{
Repeatability and validity of a field kit for estimation of cholinesterase in whole blood
}

\author{
L London, M L Thompson, S Sacks, B Fuller, O M Bachmann, J E Myers
}

\begin{abstract}
Objectives-To evaluate a spectrophotometric field kit (Test-Mate-OP) for repeatability and validity in comparison with reference laboratory methods and to model its anticipated sensitivity and specificity based on these findings.

Methods-76 farm workers between the age of 20 and 55, of whom 30 were pesticide applicators exposed to a range of organophosphates in the preceding 10 days, had blood taken for plasma cholinesterase (PCE) and erythrocyte cholinesterase (ECE) measurement by field kit or laboratory methods. Paired blinded duplicate samples were taken from subgroups in the sample to assess repeatability of laboratory and field kit methods. Field kits were also used to test venous blood in one subgroup. The variance obtained for the field kit tests was then applied to two hypothetical scenarios that used published action guidelines to model the kit's sensitivity and specificity.
\end{abstract}

Results-Repeatability for PCE was much poorer and for ECE slightly poorer than that of laboratory measures. A substantial upward bias for field kit ECE relative to laboratory measurements was found. Sensitivity of the kit to a $\mathbf{4 0} \%$ drop in PCE was $67 \%$, whereas that for ECE was $89 \%$. Specificity of the kit with no change in mean of the population was $100 \%$ for ECE and $91 \%$ for PCE.

Conclusion-Field kit ECE estimation seems to be sufficiently repeatable for surveillance activities, whereas PCE does not. Repeatability of both tests seems to be too low for use in epidemiological dose-response investigations. Further research is indicated to characterise the upward bias in ECE estimation on the kit.

(Occup Environ Med 1995;52:57-64)

Keywords: cholinesterase; field kit; repeatability

Agrichemicals are extensively used in farming and public health programmes worldwide and their potential for adverse health effects is

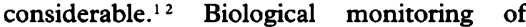
humans potentially exposed to agrichemicals has been widely recommended for the prevention of pesticide poisoning ${ }^{34}$ and for purposes of epidemiological research. ${ }^{5}$ Proposed changes to South African occupational health legislation ${ }^{6}$ are likely to make medical monitoring of workers exposed to potentially hazardous chemicals mandatory and thus bring statutory requirements into line with international standards. ${ }^{47}$ Given that farm workers will fall under the ambit of the new legislation, ${ }^{6}$ attention is likely to be directed at identifying cheap and practical methods that are both reliable and valid for field monitoring of workers exposed to agrichemicals.

Organophosphates and carbamates constitute the most widely used insecticides in South Africa at present, ${ }^{89}$ and measurement of serum and red blood cell cholinesterase is widely used to monitor exposed people..$^{1341011}$ Decreased plasma cholinesterase (PCE) activity is regarded as a marker of recent exposure, and in erythrocytes (ECE) of neuronal toxicity. ${ }^{3}$ The magnitude of cholinesterase depression is also used as an action guide for removal of a worker from further exposure. ${ }^{34}$

Laboratory methods for estimating cholinesterase activity have been well described. ${ }^{312}$ For public health purposes, however, methods of field cholinesterase estimation are advantageous. Field kits, with either tintometric or kinetic spectrophotometry, are available. $^{312}$ Studies have found that the kinetic spectrophotometry has better precision for estimation of erythrocyte cholinesterase than has the tintometer, ${ }^{12}$ particularly when standardised for haemoglobin concentration in the blood. ${ }^{13}$ This method is being used in developing countries as part of the East Africa pesticide network project ${ }^{14}$ and has been reported as being sufficiently precise to obviate the need for laboratory specimens. ${ }^{15}$ As a result of promising initial research results, the World Health Organisation is currently helping to develop the spectrophotometric method as a valid and reliable field device for estimation of cholinesterase. ${ }^{16}$

Published data on the precision and validity of such field methods are not available, and their usefulness in field studies on health effects of exposure to pesticides is still unclear. To fill some of these gaps, this study aimed to assess the repeatability and validity of the field kit for estimation of cholinesterase by the spectrophotometric method (TestMate OP kit) ${ }^{1213}$ in relation to standard laboratory methods based on the procedure of Ellman et al. ${ }^{17}$ Farm workers in the Western Cape region of South Africa provided the specimens. These data were then used to model the sensitivity and specificity of the kit in 
Figure ! Cholinesterase testing in the study sample.

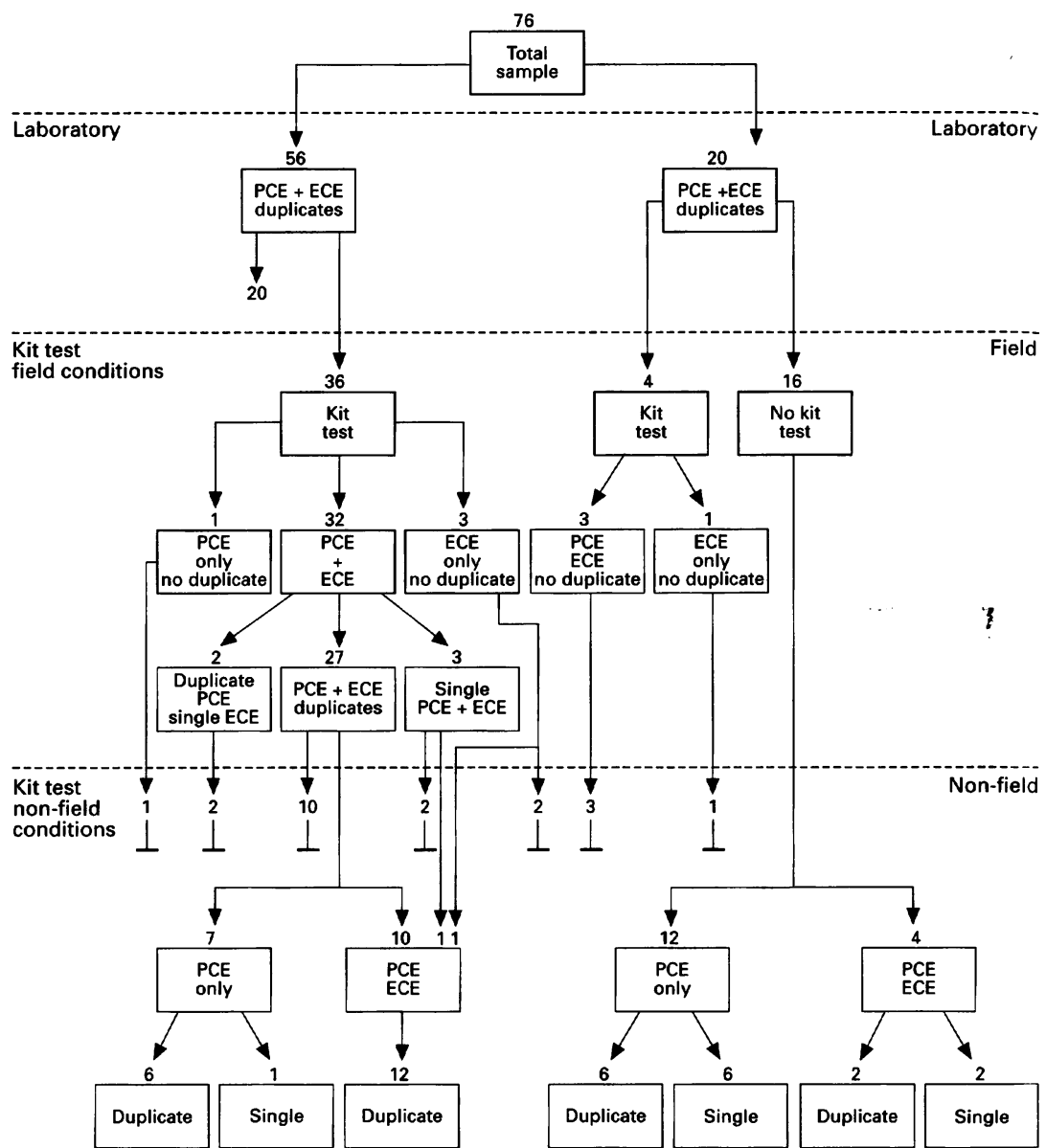

the detection of critical changes in cholinesterase activity. The study was performed as part of a larger investigation of potential long term adverse health effects arising from exposure to agrichemicals among fruit farm workers for whom laboratory estimations of PCE and ECE were being collected.

\section{Methods}

The total sample consisted of 76 subjects who received either field kit or laboratory estimations of PCE and ECE activity or both (fig 1). The sample was accumulated on three successive field trips during a period of three weeks in March 1993 and included $80 \%$ of the participants who attended for the larger neurotoxicity study already referred to. All subjects were male workers between the ages of 20 and 55 , and included 30 workers involved in agrichemical application who had been exposed to a range of organophosphates within the past 10 days.

Field procedures for blood sampling were as follows: $10 \mathrm{ml}$ of heparinised venous blood was taken from each subject for cholinesterase estimation. Fifty six subjects had a second sample taken with a false subject name for blinded laboratory repeat PCE and ECE estimations. Venous blood was kept on ice and reached the laboratory within eight hours of venepuncture. Field kit estimations were performed on $10 \mu \mathrm{l}$ of blood derived from a finger prick according to methods described by the suppliers. ${ }^{1318}$ One examiner was responsible for all field kit estimations (MB). Attention was paid to avoid excessive thermal exposure or vibration damage to the device during transport, and temperature on the collection days ranged between 20 and $28^{\circ} \mathrm{C}$.

Subgroups of subjects underwent repeat finger prick field tests (non-blinded) during the same sampling session for PCE and ECE. Also, parallel estimations of field kit PCE and ECE (duplicate and non-duplicate) were performed on heparinised venous blood from another subgroup of subjects on the same day under non-field conditions. Figure 1 shows the numbers in the groups. The differing numbers of subjects included in various tests and 
Fioure 2 Repeatability: $v$ mean field kit $P C E$. difference in field kit PCE

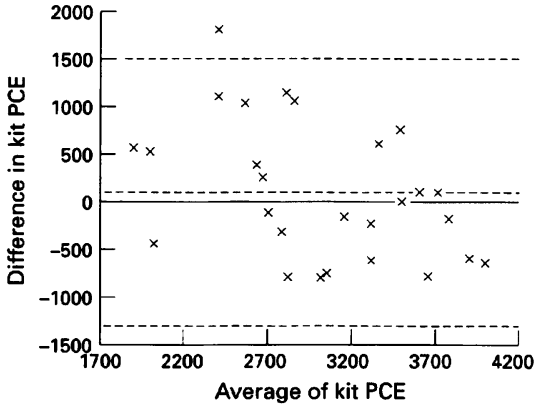

settings were the result of logistical constraints imposed by the field conditions under which data were collected as part of a larger study.

Laboratory estimation of cholinesterase was performed at the Chemical Pathology Laboratory at Groote Schuur Hospital, a large teaching hospital in Cape Town. The PCE was measured by the method of Knedel and Bottger with butyrylthiocholine as substrate ${ }^{19}$ and ECE by the method of Ellman et al ${ }^{17}$ with acetylthiocholine as substrate. Haemolysates for ECE were prepared as described previously. ${ }^{20}$ Reagents were supplied as kits (Boehringer-Mannheim) and analyses were performed at $30^{\circ} \mathrm{C}$ with an automated analyser (Hitachi 704). Commercial quality control serum samples, two each for PCE and ECE (Precipath and Precinorm, BoehringerMannheim) were run within the batches of assays for PCE and ECE and good agreement was found with the stated mean values provided by the suppliers.

Field kit estimation for PCE uses butyrylthiocholine and ECE uses acetylthiocholine as a substrate and determines cholinesterase activity on whole blood by means of a battery powered computer driven colorimeter. The ECE field kit assay makes use of an inhibitor As1397 to prevent PCE interference in whole blood (R Magnotti, personal communication, 18 June 1992). Steps in the assay are guided by digital commands, key activated functions and computer generated timing to assist reproducibility. The kit has a photometric haemoglobinometer, and performs an automated adjustment for haemoglobin

Figure 3 Repeatability: difference in laboratory $P C E$ v mean laboratory PCE. concentration as well as a correction for assay results to $25^{\circ} \mathrm{C}$ based on an inbuilt temperature sensor. ${ }^{18}$

For comparison between laboratory and kit methods, results obtained from the kit for PCE were corrected from $25^{\circ}$ to $30^{\circ} \mathrm{C}$ by multiplying kit results by a temperature factor of 1.24 and by a factor of 1000 to adjust from $\mathrm{U} / \mathrm{ml}$ to $\mathrm{U} / \mathrm{h}$, and multiplying laboratory results by (1-packed cell volume) to adjust to measurement on whole blood. For ECE, results were standardised by haemoglobin concentration,,$^{13}$ and kit estimates were corrected to $30^{\circ} \mathrm{C}$ by multiplying by a factor of 1.28. Results for PCE (U/1) and ECE (U/g haemoglobin) are therefore given corrected to $30^{\circ} \mathrm{C}$.

\section{ANALYSIS}

Three methods of determination of PCE and ECE are compared in this study: field application of the kit, non-field application of the kit, and laboratory. Repeatability of each methpd was assessed by comparing duplicate measurements taken with the same method (field kit, kit under non-field conditions, or laboratory) and kit (field or non-field) validity was assessed by comparing kit measures with those from the laboratory on the same subjects.

The statistical assessment of repeatability and validity has been discussed in some detail by Bland and Altman. ${ }^{21}$ For each assessment, the Pearson's correlation coefficient was determined to establish the degree of linear agreement between the two measures under consideration (repeat measures under one method for reliability or comparison of one method with another for validity). The Pearson statistic was used after having first confirmed normality of the distributions. Plots were also constructed of the difference in the two measurements $v$ their average. ${ }^{21}$ Such plots enable one to graphically display the variability across repeat measurements and to identify any tendency for that variability to change with activity. For reasons of brevity, only the plots for repeatability and validity of PCE are presented.

The mean (SD) - that is, the bias - of the differences were also determined. The SD of the difference across duplicate measurements with the same method provides an estimate of the repeatability of that method. Two SDs is the definition of a repeatability coefficient adopted by the British Standards Institution, ${ }^{22}$ and this was used to calculate a lower limit (mean difference -2 SD) and an upper limit (mean difference +2 SD) of agreement. The use of the $\mathrm{SD}$ as a measure of variability requires approximate normality of the differences, which was verified in each case. Analyses were carried out with the SAS and Statgraphics statistical packages. ${ }^{23} 24$

Comparison was then made to recognised activity standards for changes in cholinesterase activities ${ }^{34}$ to assess how well the kit would perform as a monitoring tool. Based on the variability of kit results, and with the baselines obtained in the study for PCE and ECE, the probability was estimated of missing a 
Table 1 Repeatability of cholinesterase measurements

\begin{tabular}{|c|c|c|c|c|c|c|}
\hline Method $^{\star} n$ & Mean & Correlationt & Mean difference $(95 \%$ CI) $\ddagger$ & Limits of & agreement & $5 \% C I) \oint$ \\
\hline \multicolumn{7}{|l|}{ PCE: } \\
\hline FK 29 & $3091 \cdot 2$ & 0.59 & $95 \cdot 3(-172 \cdot 2$ to $362 \cdot 8)$ & $\begin{array}{l}\text { Lower } \\
\text { Upper }\end{array}$ & $\begin{array}{r}-1311 \cdot 6 \\
1502 \cdot 2\end{array}$ & $\begin{array}{l}(-1774.9 \text { to }-848 \cdot 2) \\
(1038.8 \text { to } 1965.5)\end{array}$ \\
\hline NFK 26 & $3561 \cdot 2$ & 0.84 & $3.8(-208.9$ to 216.5$)$ & $\begin{array}{l}\text { Lower } \\
\text { Upper }\end{array}$ & $\begin{array}{r}-1049 \cdot 2 \\
1056 \cdot 8\end{array}$ & $\begin{array}{l}(-1417 \cdot 7 \text { to }-680 \cdot 8) \\
(688.4 \text { to } 1425 \cdot 3)\end{array}$ \\
\hline Lab 56 & $3374 \cdot 9$ & 0.97 & $-11.6(-53.5$ to 30.3$)$ & $\begin{array}{l}\text { Lower } \\
\text { Upper }\end{array}$ & $\begin{array}{r}-331 \cdot 7 \\
308 \cdot 5\end{array}$ & $\begin{array}{l}(-404 \cdot 3 \text { to }-259 \cdot 1) \\
(235.9 \text { to } 381 \cdot 1)\end{array}$ \\
\hline \multicolumn{7}{|l|}{ ECE: } \\
\hline FK 27 & $45 \cdot 1$ & 0.85 & $1 \cdot 3(-0 \cdot 10$ to $2 \cdot 70)$ & $\begin{array}{l}\text { Lower } \\
\text { Upper }\end{array}$ & $\begin{array}{r}-5 \cdot 76 \\
8 \cdot 36\end{array}$ & $\begin{array}{l}(-8.18 \text { to }-3.34) \\
(5.94 \text { to } 10.78)\end{array}$ \\
\hline NFK 14 & $45 \cdot 7$ & 0.32 & $1.3(-1.64$ to 4.24$)$ & $\begin{array}{l}\text { Lower } \\
\text { Upper }\end{array}$ & $\begin{array}{r}-8 \cdot 90 \\
11 \cdot 50\end{array}$ & $\begin{array}{l}(-14.00 \text { to }-3.80) \\
(6.40 \text { to } 16 \cdot 60)\end{array}$ \\
\hline Lab 56 & $36 \cdot 0$ & 0.96 & $-0.1(-0.48$ to 0.28$)$ & $\begin{array}{l}\text { Lower } \\
\text { Upper }\end{array}$ & $\begin{array}{r}-2 \cdot 91 \\
2 \cdot 89\end{array}$ & $\begin{array}{l}(-3.66 \text { to }-2 \cdot 34) \\
(2.14 \text { to } 3.46)\end{array}$ \\
\hline
\end{tabular}

$\star$ FK = field kit; NFK = non-field kit; Lab = laboratory.

† Pearson's correlation between repeat measures.

† Average difference across repeat measures.

₹ Average difference across repeat measures.

Upper limit = mean difference between repeat measures plus 2 SDs of the difference between repeat measures.

$30 \%$ drop in baseline cholinesterase in the presence of a true drop of $40 \%$ (false negatives), as well the probability of finding an apparent $30 \%$ drop in the presence of an unchanged "true" baseline (false positives) for both PCE and ECE.

\section{Results}

REPEATABIITYY

\section{$P C E$ determinations}

Figures 2 and 3 show plots of the difference in the duplicate PCE measurements $v$ their average for the field kit and laboratory respectively, and indicate graphically the limits of agreement, and the mean difference based on repeat measures. ${ }^{21}$ Table 1 shows these estimates with $95 \%$ confidence intervals $(95 \%$ CIs), and the correlation coefficients based on repeat measures.

As is to be anticipated from duplicate measures, there is essentially no bias (the $95 \% \mathrm{CIs}$ for all mean differences include 0 ), but the repeatability of the laboratory measurements of PCE is clearly far better than that of the field kit, with a much narrower $95 \% \mathrm{CI}$ in the mean difference for laboratory measures. This is shown in the greater scatter of results of PCE estimations found for the kit (fig 2) than for the laboratory (fig 3). No trend in differences was found across a range of PCE measurements for either kit or laboratory measures.

The PCE repeatability coefficient (implicit in table 1 as the upper limit of agreement less the mean difference) for the field kit is 4.4 times greater than the repeatability coefficient for the laboratory and 1.3 times that of the kit under non-field conditions. This suggests worse agreement for repeat field kit PCE measurements relative to laboratory measurements, with some improvement when the kit is used under non-field rather than field conditions.

\section{ECE determinations}

Table 1 shows the estimates of the correlation between repeated measures of ECE on the field kit and in the laboratory, the mean differences, and the upper and lower limits of agreement.

Once again, the repeatability of the laboratory measurements is far better than that of both the field and non-field application of the kit. In this case, the repeatability of the nonfield kit is worse than that of the field kit, but this is based on a small sample $(n=14)$. The repeatability coefficient of the field kit ECE, implicit in table 1 as the upper limit of agreement less the mean difference, is 2.4 times that of the laboratory. Although the field kit ECE still performed worse than the laboratory, this represents an improvement in quality when compared with the PCE repeatability.

\section{VALIDITY}

PCE determinations

Figure 4 shows a plot of the difference between kit (under field conditions) and laboratory PCE measurements $v$ their average,

Table 2 Validity of cholinesterase measurements

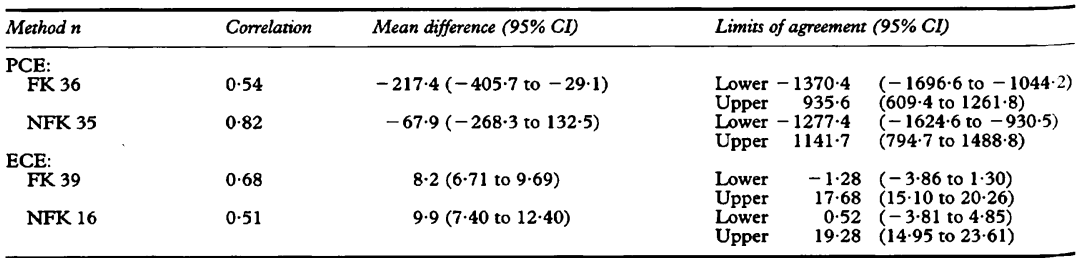

Footnotes as for table 1 
Figure 4 Validity: difference between field kit difference average of field kit and laboratory PCE.

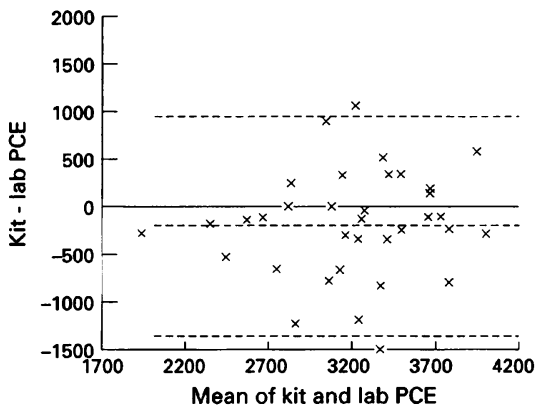

and graphically illustrates the mean difference and the limits of agreement. Again, a wide scatter is evident, with a slight downward bias of kit relative to laboratory. This is confirmed in table 2 , which summarises data on the difference between laboratory, field, and nonfield kit PCE measurements and provides estimates of correlation, bias, and upper and lower limits of agreement.

The downward bias of the kit measurements relative to the laboratory measurements is not substantial relative to the activity of PCE. For example, the mean difference between field kit and laboratory measures is of the order of $6 \%$ of mean laboratory PCE in table 1, and for non-field use of the kit the $95 \% \mathrm{CI}$ of the differences includes 0 . In absolute terms, field kit PCE may be from $1370.3 \mathrm{U} / 1$ below to $935.6 \mathrm{U} / 1$ above the laboratory readings for the same subject. The nonfield kit application performs better than the field kit in terms of bias, but they are of comparable variability relative to the laboratory measurements.

\section{ECE determinations}

Table 2 shows analagous estimates of correlation, bias, and upper and lower limits of agreement for ECE measurements that compare laboratory methods with field and nonfield kit determinations. The kit is biased upwards and, in contrast to PCE, the extent of the bias relative to the laboratory ECE activities is substantial. Field kit ECE may be $1.3 \mathrm{U} / \mathrm{g}$ haemoglobin below to $17.7 \mathrm{U} / \mathrm{g}$ haemoglobin above the laboratory readings and the mean difference between kit and laboratory measures is of the order of $23 \%$ of mean laboratory ECE. The variability of the field and non-field application of the kit relative to the laboratory is comparable.

Table 3 Probability of false negatives and false positives in detecting a decrease in cholinesterase activity

\begin{tabular}{lll}
\hline & False negative $^{*}$ & False positivet \\
\hline PCE & 0.33 & 0.09 \\
ECE & 0.11 & 0.00
\end{tabular}

^False negative $=$ a person's measurement after exposure is $>70 \%$ of his or her measurement before exposure when, in $>70 \%$ of his or her measurement before exposure when, reality, the population mean concentrations are $40 \%$ lower. $+F a l s e$ positive $=$ a person's measurement after exposure is
$<70 \%$ of his or her measurement before exposure when, in $<7 \%$ of his or her measurement before exposure when, in
reality, the population mean concentrations are unchanged.
Table 3 shows the estimates of probabilities of false positives and false negatives calculated from the variances estimated from repeat readings in our sample with an assumed 30\% drop in baseline cholinesterase as a threshold for action. For PCE a $30 \%$ drop in activities from the observed field kit average of $3091 \cdot 2$ $\mathrm{U} / \mathrm{l}$ would be $927 \cdot 4 \mathrm{U} / \mathrm{l}$ and for ECE (average $45.1 \mathrm{U} / \mathrm{g}$ ) would be $13.5 \mathrm{U} / \mathrm{g}$. The kit would miss a true drop in PCE of $40 \%$ from baseline in $33 \%$ of cases, and a $40 \%$ drop in ECE in $11 \%$. A full exposition of the method of calculation is contained in the appendix.

\section{Discussion}

Data from this study suggest limitations to the validity and repeatability of the field kit relative to laboratory measurements. The repeatability of PCE and ECE of the kit seems much poorer than that of the laboratory (table 1) and for PCE, this is probably a conservative assessment. The adjustment of laboratory results for PCE described earlier involved? second measured variable (packed cell volume) that is subject to its own variability. The combination of two measured variables, each with its own spread, will increase the variability of the adjusted laboratory measures and overestimate the laboratory repeatability coefficient, with the result that the true picture may be worse than that reflected in this study for PCE.

Poor kit repeatability for PCE and ECE seems to be partly a feature of the kit itself. We would attribute this to a number of possible factors-for example, the field kit is operated by non-laboratory personnel and is subject to changes in ambient temperature, vibration and movement, and other environmental variables, as well as being subject to internal instrument error. In contrast, the separation and washing steps required for laboratory ECE measurement are done by trained technicians and the laboratory analyser operates in a controlled environment, has strict temperature and voltage control, and uses fully automated pipetting and mixing steps.

Even when venous blood was used as the source for kit estimations (non-field conditions), which may be expected to obviate many of the field obstacles to optimal test performance, (including haemoconcentration in successive drops of finger prick blood), the repeatability of the kit PCE determinations improved somewhat, but remained substantially worse than that of the laboratory. It must also be borne in mind that the kit is intended for use in field conditions and that this is where its usefulness most needs to be evaluated.

The validity assessment in this study suggested that relative to the laboratory, the field kit readings of PCE were systematically low, and that there was also a more substantial upward bias relative to the laboratory in the kit ECE readings. In the examination of plots of difference against the mean $^{21}$ no evidence was present that the 
extent of the bias varied across the range of cholinesterase readings.

Clearly, the kit and laboratory results for ECE were not comparable. One explanation for the results may lie in the different manner in which interference by PCE in the measurement of ECE is prevented. In the laboratory, this is accomplished by physical separation of plasma and erythrocytes, whereas the kit relies on As1397 to inhibit PCE in whole blood. Incomplete inhibition of the PCE in the field kit may be responsible for the overestimation of ECE. Another explanation may be the presence of residual acetylcholinesterase activity in serum, ${ }^{25}$ reported to be of the order of $10 \% .{ }^{26}$ If either of these factors are constant across a range of ECE activities, as suggested by our data, it may be possible to adjust for this overestimation by applying a conversion factor, and further research is needed to clarify this possibility.

A different approach to validity is to establish normal ranges for the field kit based on appropriate reference populations. However, data on this has yet to be reported in the scientific literature, and the difficulties of locating an appropriate control group, particularly in South Africa, are considerable. High levels of alcohol consumption, chronic liver disease, malnutrition, and anaemia are known determinants of PCE and ECE activities ${ }^{3}$ and are factors that are prevalent among both farm workers and non-farm worker populations in South Africa. Also, the possibility of environmental contamination due to agrichemicals in rural areas would further complicate the use of rural control subjects to establish normal ranges.

A preferred method of estimation of cholinesterase to identify affected workers is to use subjects as their own controls in prospective measurements over a season, and this is recommended in the field kit manual. ${ }^{18}$ The comparison of a cholinesterase result with a subject's baseline value before exposure is the object of biological monitoring in the workplace to enable prompt preventive action to be taken. California regulations rely on a decrease from baseline activities of $30 \%$ of either PCE or ECE to warrant further action (repeat test) and of $40 \%$ in ECE and $50 \%$ in PCE to spur removal from exposure. ${ }^{3}$

Based on the repeatability found in this study, and with the observed baselines (table 3 ), the kit seems to miss a true drop in PCE of $40 \%$ from baseline in $33 \%$ of cases. The equivalent probability for a $40 \%$ drop in ECE is $11 \%$, which is more satisfactory. The poor performance of the kit PCE may be partly due to exposed study subjects who have PCE activities already lower than normal. From the same calculations as in the appendix, but with higher baselines for PCE-that is, 4000 and 6000 -the frequency of false negatives drops to $29 \%$ and $20 \%$ respectively, but still remains unacceptably high.

Moreover, these results reflect an optimistic assessment as they are based on measurements taken at a single point in time. Greater variability would be introduced with addi- tional measurements at different points in time, which would therefore decrease the likelihood of detecting real changes in cholinesterase activities. Follow up data are presently being analysed to illucidate the effects of this added variability on the ability of the kit to detect changes in PCE and ECE over time.

High levels of instrument variability also compromise the usefulness of the kit in epidemiological studies to investigate dose response relations. This is exacerbated where within and between individual variation in the measured variable may be high, as has been reported for cholinesterase activity. ${ }^{3}$ Estimates of maximum variation in PCE activity of healthy subjects are of the order of $9 \%{ }^{3}$ to $14 \%$, ${ }^{27}$ whereas that for between person variability may be substantially higher. ${ }^{328}$ If a measurement device itself has poor repeatability, the chances of non-differential misclassification are greatly enhanced, and the value of the field kit with a repeatability coefficient as high as shown by our data, may render the kit of limited value for detecting exposure-outcome effects. This served as an important motivation for a decision to rely on laboratory measures of cholinesterase in our larger study of chronic neurotoxic effects.

An issue not assessed in this study is the effect of different readers on kit repeatability and validity as all kit measurements were performed by a single observer (MB). Given that one researcher performed all the kit tests in a standardised manner, we think that noninstrument human error was kept to a minimum in this study. This is consistent with our experience in piloting the device in 1992 where a different reader (LL) found a similar upward bias in kit ECE and poorer repeatability for both kit ECE and PCE relative to the laboratory, although the levels of variability of the methods differed from those of the current study.

An additional reason for the use of field monitoring devices may be economic. In our experience, however, in a developing country, the costs of the field kit tests are not insubstantial when compared with those of laboratory tests. For example, laboratory costs for PCE and ECE assays are about $\$ 4$ and \$5-20 respectively, whereas the costs of purchasing the kit and reagents for the first 200 PCE and ECE assays were of a similar order (\$5). With increasing numbers of tests, the average costs for the field kit decrease because of economy of scale. Unless the kit is to be used for a large number of tests, this cost saving will not be substantial.

\section{Conclusion}

Despite the undoubted value of a field instrument, to be used on farms to obtain immediate cholinesterase results with minimal reader training, limitations are apparent in the performance of the field kit for cholinesterase measurement relative to laboratory measurements. These include the presence of an upward bias of ECE as measured on the kit 
relative to the laboratory, and more importantly, poor kit repeatability relative to laboratory measurements, particularly for PCE.

These shortcomings seriously detract from the potential usefulness of the kit for epidemiological studies to investigate dose response relations, and for surveillance purposes. Because the ECE repeatability of the field kit is somewhat better than PCE, the estimation of ECE by the field kit may be sufficient for purposes of biological monitoring with the use of a model based on California regulations. The ease and simplicity of use of the kit needs to be balanced against its lack of repeatability in the field.

Several issues for future research into the kit have been raised in this study. These include the characterisation of the constancy of the upward bias in ECE estimation and the reasons for this bias, the elaboration of the sources of variation that contributed to poor repeatability of the kit results, and field research into the impact of less than ideal field conditions on kit performance, which is especially important for its use in developing countries where field methods are most needed. ${ }^{29}$ In particular, the effect of multiple observers and instruments remains unexplored, and the use of slightly different observer technique in giving the test may play an important part in adding to the variability inherent in the instrument. It would be particularly useful if studies sought to evaluate and validate techniques for assay performance, particularly given that the prime objective of the kit is for field use where robustness of the methods is essential.

This study was supported by a research grant from the International Development Research Centre, the Guy Elliot Research Fellowship in the Department of Medicine at the University of Cape Town and the South African Medical Research Council. Acknowledgement is made of the enthusiastic participation and cooperation of the farm workers, farmers, this study.

\section{Appendix: Estimation of false positives and false} negatives in cholinesterase tests with the field kit

Let the baseline measurement (of PCE or ECE) for a person in a particular population be $X_{1}$ and the subsequent measurement for that person be $\mathrm{X}_{2}$, and assume that $X_{i} \sim N\left(\mu_{i}, \sigma_{i}^{2}\right), i=1,2$. Assume further that $\mathbf{X}_{1}-\mathbf{X}_{2} \sim \mathrm{N}\left(\mu_{\text {diff }}, \sigma_{\text {diff }}{ }^{2}\right)$

The threshold for action with regard to a person is a drop in cholinesterase to below $70 \%$ of the baseline measure. Thus if $\mathrm{X}_{2}<0.7 \mathrm{X}_{1}$ then further action is indicated.

False positives occur if:

$\mathrm{X}_{2}<0.7 \mathrm{X}_{1}$ but, in fact, $\mu_{\text {diff }}=0$.

False negatives occur if:

$\mathrm{X}_{2} \geqslant 0.7 \mathrm{X}_{1}$ but, in fact, $\mu_{\text {dif }}=\mathrm{c}$, where $\mathrm{c} \geqslant 0.3 \mu_{1}$.

To be able to estimate the sensitivity and specificity of this action threshold, we use the sample values of the various model parameters. Specifically, we assume for PCE that:

$\begin{array}{llr}\mu_{1} & =3140.0 \\ \sigma_{1} & 589.7 \\ \sigma_{2} & 863.4 \\ \sigma_{\text {dif }} & =703.4 \\ \operatorname{cov}\left(X_{1}, X_{2}\right) & 300396.7\end{array}$

It must be kept in mind, however, that $\sigma_{\text {diff }}{ }^{2}$ and $\operatorname{cov}\left(\mathbf{X}_{1}, \mathbf{X}_{2}\right)$ are not based on repeat measurements separated by a period of time-for example: before and after the season, and so we are almost certainly underestimating the variability and hence giving too optimistic an assessment of the usefulness of the field kit in this context.

The probability of action being signalled is then given by:

$P\left(X_{2}<0.7 X_{1}\right)$ or $\mathbf{P}\left(0.7 X_{1}-X_{2}>0\right)$.

Now $\quad 0.7 \mathrm{X}_{1}-\mathrm{X}_{2} \sim \mathrm{N}\left(\mu_{\mathrm{diff}}-0.3 \mu_{1}, \quad \sigma_{\mathrm{diff}}{ }^{2}-0.51\right.$ $\left.\sigma_{1}^{2}+0.6 \operatorname{cov}\left(X_{1}, X_{2}\right)\right)$.

Under the null hypothesis that $\mu_{\text {dif }}=0$, we have that:

$\mathrm{P}\left(\mathrm{X}_{2}<0.7 \mathrm{X}_{1}\right)=1-\mathrm{P}\left(\mathrm{Z}_{\mathrm{N}(0,1)}<1.34\right)=0.09$.

Thus we estimate that if there is no change in mean PCE activity, $9 \%$ of workers will be falsely diagnosed as having PCE activities that have fallen below the threshold. This means that the specificity of this PCE action procedure, when applied through the field kit, is $91 \%$. It must be kept in mind that this may be an optimistic assessment.

We consider further the proportion of subjects who would not be detected by this action procedure in the situation where the true mean PCE activities have dropped by $40 \%$. Under the alternative hypothesis that $\mu_{\text {diff }}=0.4 \mu_{1}$, we have:

$P\left(X_{2} \geqslant 0.7 X_{1}\right)=P\left(Z_{x(0,1)} \leqslant-0.45\right)=0.33$

Thus if the true mean PCE activity of the subjects has, in fact, dropped by $40 \%, 33 \%$ of such subjects will not be detected by this action threshold. This means that the sensitivity of the PCE action procedure (in the presence of a $40 \%$ drop in PCE activity) is $67 \%$.

The calculations for the field kit ECE follow similarly, where we assume (again with sample values) that:

$$
\begin{array}{lll}
\mu_{1} & =45.5 \\
\sigma_{1} & =6.42 \\
\sigma_{2} & =6.68 \\
\sigma_{\text {diff }} & =3.53 \\
\operatorname{cov}\left(\mathrm{X}_{1}, \mathrm{X}_{2}\right) & =36.624
\end{array}
$$

Table 3 shows these results.

1 World Health Organisation. Public health impact of pesticides used in agriculture. Geneva; WHO, 1990.

2 Jeyaratnam J. Health problems of pesticide usage in the third world. Br f Ind Med 1985;42:505-6.

3 Coye MJ, Lowe JA, Maddy KT. Biological monitoring of agricultural workers exposed to pesticides: I. cholinesterase activity determinations. f Occup Med 1986;28 619-36.

4 Ames RG, Brown SK, Mengle DC, Kahn E, Stratton JW, Jackson RJ. Protecting agricultural applicators from overexposure to cholinesterase-inhibiting pesticides: perspectives from the California programme. Fournal of the Society of Occupational Medicine 1989;39:85-92. 5 Hulka BS, Margolin BH. Methodological issues in Epidemiol 1992;135:200-9.

6 Department of Manpower. Occupational Safety and Health Act 85/93. Pretoria: Government Printers, 1992.

7 Ministry of Agriculture, Fisheries, and Food (MAFF) and the Health and Safety Commission (HSC). Pesticides: code of practice for the safe use of pesticides on farms and code of practice for the safe use of
holdings. London: HMSO, 1990.

8 London L, Myers JE. Quantifying pesticide hazards in the agricultural sector in the Western Cape 1989. Cape Town: Department of Community Health, University of Cape Town, Observatory, 1992. (Departmental of Cape Town

9 London L, Myers JE. Critical issues in agrichemical safety in South Africa. $A m \mathcal{f}$ Ind Med 1994 (in press).

10 Knaak JB, Maddy KT, Jackson T, Frederickson AS, Peoples SA, Love $R$. Cholinesterase activity in blood samples collected from field workers and nonfield workers in California. Toxicol Appl Pharmacol 1978;45:755-70.

11 Kangas J, Jauhinen A. Determination of cholinesterase activity. African Newsletter on Occupational Health and Safety 1991;2:56-8.

12 Magnotti RA, Dowling K, Eberly P, McConnell RS. Field measurement of plasma and erythrocyte cholinesterases. Clin Chi Acta 1988;176:315-32.

13 McConnell RS, Cedillio L, Keifer M, Palomo MR Monitoring organophosphate insecticide-exposed workers for cholinesterase depression: new technology for 
office or field use. Foumal of Occupational Medicine of fanan 1992;34:34-7.

14 Kangas J, Jauhiainen A. Determination of cholinesterase Newsletter on Occupational Health and Safety 1991;2:56-8.

15 Richter ED, Chuwers P, Levy Y, Gordon M, Grauer F, Marzouk J, et al. Health effects from exposure to organophosphate pesticides in workers and residents in Israel. Isr $f$ Med Sci 1992;28:584-97.

16 International programme on chemical safety (IPCS). Technical workplan of the IPCS for the period 1993-1994. Technical workplan of the IPCS for the period

17 Ellman GL, Courtney KD, Andres V, Featherstone RM. A new and rapid colourimetric determination of acetyl-
cholinesterase activity. Biochem Pharmacol 1961;7: 88-95.

18 Test-Mate OP Kit for the field determination of organophosphate pesticide exposure. Instruction book IB-15. Cincinnati: EQM Research, 1991.

19 Knedel M, Bottger R. Eine kinetische methode zur bestimmung der aktiwitat der pseudocholinesterase. (A kinetic method for determining pseudocholinesterase). Klin Wochenschr 1967;45:325-7.

20 Lewis PJ, Lowing RK, Gompertz D. Automated discrete kinetic method for erythrocyte acetylcholinesterase and plasma cholinesterase. Clin Chem 1981;27:926-9.

21 Bland JM, Altman DG. Statistical methods for asses- sing agreement between two methods of clinical mea. surement. Lancet 1986;8476:307-10.

22 British Standards Institution. Precision of test methods I guide for the determmation and reproducibility for a standard test method. London: BSI, 1979. (BS 5497, part I.)

23 SAS Institute SASISTAT User's guide, version 6.08. 4th ed Cary: SAS Institute 1989.

24 Statistical Graphics Corporation. Statgraphics user manual. Version 6. Manugistics. Cambridge: SGC, 1992.

25 Pilz W. Cholinesterases. In: Bergmeyer HU, ed. Methods of enzymatic analysis. New York: Verlag Chemie Weinheim enzymatic analysis. New
Academic Press, 1974.

26 Lotti $M$. Central neurotoxicity and behavioural effects of anticholinesterases. In: Ballantyne B, Marrs TM,
Aldridge $\mathrm{WN}$, eds. Clinical and experimental toxico. Aldridge WN, eds. Clinical and experimental toxicology of organophosph

27 Brock A. Inter and intraindividual variations in plasma cholinesterase activity and substance concentration in employees of an organophosphorus insecticide factory. Br f Ind Med 1991;48:562-7.

28 Lander F, Lings $S$. Variation in plasma cholinesterase activity among greenhouse workers, fruitgrowers and slaughtermen. Br f Ind Med 1991;48:164-6.

29 Partanen T, Kurppa K, Ngowi VF. Occupational pesticide hazards in developing countries: epidemiological considerations. African Newsletter on Occupational Health and Safety 1991;2:46-51. 